\title{
Thymidylate synthase expression and molecular alterations in adenosquamous carcinoma of the lung
}

\author{
Catherine Shu ${ }^{1}$, Haiying Cheng ${ }^{2}$, Antai Wang ${ }^{3}$, Mahesh M Mansukhani ${ }^{4}$, Charles A Powell ${ }^{5}$, \\ Balazs Halmos ${ }^{2}$ and Alain C Borczuk ${ }^{4}$ \\ ${ }^{1}$ Department of Internal Medicine, New York Presbyterian Hospital-Columbia University Medical Center, \\ New York, NY, USA; ${ }^{2}$ Division of Hematology/Oncology, Herbert Irving Comprehensive Cancer Center, \\ New York Presbyterian Hospital-Columbia University Medical Center, New York, NY, USA; ${ }^{3}$ Department of \\ Biostatistics, Herbert Irving Comprehensive Cancer Center, New York Presbyterian Hospital-Columbia \\ University Medical Center, New York, NY, USA; ${ }^{4}$ Department of Pathology and Cell Biology, New York \\ Presbyterian Hospital-Columbia University Medical Center, New York, NY, USA and ${ }^{5}$ Division of Pulmonary, \\ Critical Care and Sleep Medicine, Mount Sinai School of Medicine, New York, NY, USA
}

\begin{abstract}
Thymidylate synthase expression is known to be higher in squamous cell carcinoma than in adenocarcinoma of the lung. It is thought that this is the reason for the poor efficacy of pemetrexed in squamous cell carcinoma. However, there is limited data on thymidylate synthase expression in adenosquamous carcinoma, a distinct subtype of lung cancer containing both squamous and glandular differentiation. Furthermore, molecular alterations like epidermal growth factor receptor and Kirsten rat sarcoma 2 viral oncogene homolog mutations, which are seen in adenocarcinomas, are not well understood in mixed histology tumors such as adenosquamous carcinoma. In our study, we sought to better characterize adenosquamous tumors of the lung. Using immunohistochemistry to evaluate thymidylate synthase protein levels, we found that the expression of thymidylate synthase in these mixed tumors roughly parallel that of squamous cell carcinoma, instead of falling in between squamous cell and adenocarcinoma. Of note, in adenosquamous samples, the expression of thymidylate synthase was more closely correlated within the two components than would be expected by random chance alone. Also, we had a relatively high rate of epidermal growth factor receptor (11\%) and Kirsten rat sarcoma 2 viral oncogene homolog $(33 \%)$ mutations in these specimens, with the mutations showing convergence in both the glandular and squamous components upon microdissection. Our results indicate that adenosquamous carcinomas are not simple mixtures of their two histological components; they rather behave as their own entity, and it is important to further understand their behavior. Given the similarity of thymidylate synthase expression between squamous cell and adenosquamous carcinoma, and that thymidylate synthase is the main target of pemetrexed, we extrapolate that pemetrexed may also have inferior clinical activity in adenosquamous carcinoma.
\end{abstract}

Modern Pathology (2013) 26, 239-246; doi:10.1038/modpathol.2012.158; published online 21 September 2012

Keywords: adenosquamous; EGFR; KRAS; lung cancer; thymidylate synthase

Lung cancer is the second most common form of cancer in both men and women, next to prostate and breast, respectively, but is by far the leading cause of cancer death. In the United States alone in 2011, the North American Association of Central Cancer

Correspondence: Dr AC Borczuk, MD, Department of Pathology and Cell Biology, Columbia University Medical Center, 630 West 168th Street VC14-237, New York, NY 10032, USA.

E-mail: ab748@columbia.edu

Received 13 June 2012; revised 23 July 2012; accepted 24 July 2012; published online 21 September 2012
Registries estimated 239320 new diagnoses of lung cancer and 161250 deaths. ${ }^{1}$ Lung cancer can be classified histologically, with the majority being either small-cell lung cancer or non-small cell lung cancer. Together, these two account for $>98 \%$ of lung cancer diagnoses. Non-small cell lung cancer can be broken down further into three main subtypes: adenocarcinoma, large cell carcinoma, and squamous cell carcinoma. Although these account for most of the non-small cell lung cancers, some tumors are more difficult to classify and show characteristics of two or more histologies. Increasing attention to 
histological subtyping of adenocarcinoma and squamous cell carcinoma has underscored this problem, and as a result has called attention to the mixed differentiation subtypes of adenosquamous carcinoma, which currently accounts for $1 \%$ of invasive lung cancers. ${ }^{2}$ It is possible that the addition of markers for the determination of adenocarcinoma and squamous cell carcinoma will impact the frequency of adenosquamous carcinoma. Adenosquamous carcinoma is defined as a mixed tumor type, composed of both adeno (glandular) and squamous cell components, with each one comprising at least $10 \%$ of the whole tumor. ${ }^{3}$ The molecular reasons for mixed differentiation patterns remain unclear, but include a common cancer stem cell origin of the different histotypes in these tumors. Some studies have reported that the prognosis of patients with adenosquamous tumors is poorer than other non-small cell carcinomas of the lung. ${ }^{4,5}$

Up until recently, standard of care for advanced non-small cell lung cancers has been doublet chemotherapy without consideration to specific tumor characteristics aside from those criteria that impact staging. In the past few years, significant data have emerged suggesting that treatment responsiveness and toxicities may be critically dependent on both histology and molecular characteristics. For example, the anti-vascular endothelial growth factor monoclonal antibody, bevacizumab, is contraindicated in patients with squamous cell histology due to a high risk of hemoptysis and is approved only for patients with advanced non-squamous non-small cell lung cancers. ${ }^{6,7}$ Also, with the advent of epidermal growth factor receptor antagonists, such as gefitinib and erlotinib, standard regimens are being radically altered. ${ }^{8}$ These drugs seem to be uniquely effective in patients with EGFR-mutated tumors, and EGFR gene mutations are extremely rare in squamous cell tumors. Maemondo et $a l^{9}$ examined patients with advanced non-small cell lung cancer who carried EGFR mutations and found that first-line gefitinib significantly improved progression-free survival, as compared with carboplatin and paclitaxel. There is now similar promising research regarding the use of anaplastic lymphoma kinase inhibitors in patients with tumors harboring oncogenic fusions affecting $A L K{ }^{10}$

The world of traditional chemotherapy is changing as well. Pemetrexed (Alimta, Eli Lilly and Company, Indianapolis, IN, USA) is a multitargeted antifolate agent, which inhibits at least three enzymes involved in DNA synthesis: primarily thymidylate synthase, but also dihydrofolate reductase and glycinamide ribonucleotide formyl transferase. ${ }^{11}$ Hanna et $a 1^{12}$ conducted a phase III trial of pemetrexed versus docetaxel in non-small cell lung cancer patients previously treated with chemotherapy and found similar clinical efficacy outcomes, but importantly, pemetrexed led to significantly fewer side effects. A subsequent phase III study by Scagliotti et $a l^{13}$ compared cisplatin plus gemcitabine with cisplatin plus pemetrexed in chemotherapy-naive patients with advanced non-small cell lung cancer. They found that the overall survival for cisplatin/pemetrexed was non-inferior to cisplatin/gemcitabine, and interestingly, in a preplanned subset analysis, overall survival was statistically superior in the cisplatin/pemetrexed arm versus cisplatin/gemcitabine for patients with adenocarcinomas, and the opposite was noted for patients with squamous cell histology. Finally, a recent pivotal phase III study showed improved overall survival with maintenance pemetrexed therapy following four cycles of platinum-based first-line chemotherapy in patients with advanced non-small cell lung cancer only in the non-squamous as opposed to the squamous subset. ${ }^{14}$ Squamous cell carcinomas are known to have higher levels of thymidylate synthase expression than adenocarcinomas, ${ }^{15}$ and it is speculated that the efficacy of pemetrexed partially depends upon the level of thymidylate synthase expression. ${ }^{16-18}$ Accordingly, pemetrexed is approved solely for the treatment of patients with non-squamous tumors for whom it is viewed as a superior drug as compared with taxanes or gemcitabine based on the above data.

With all these new areas of research and subsequent shifts in practice, it becomes even more important to understand the mixed histology tumors. These tumors have not yet been extensively studied, and to date, no standard of care exists for treatment of adenosquamous carcinomas. Furthermore, thymidylate synthase expression levels in the different histological components of adenosquamous tumors have not been elucidated. It is unclear whether or not the same thymidylate synthase expression differential exists for these components as for the pure adenocarcinomas and squamous cell carcinomas. Given early data on mutations for EGFR and KRAS in adenosquamous carcinoma mimicking the frequency in adenocarcinoma, ${ }^{19}$ the question of whether pemetrexed responsiveness follows the genetics or the differentiation is a critical one in this subset. With this data also comes the concern for mutational heterogeneity within the tumor. Thus, the primary objective of this study was to expand on our understanding of adenosquamous carcinomas by establishing thymidylate synthase expression levels in adenosquamous carcinoma with correlation for molecular alterations, in both the overall tumor and the individual glandular/ squamous components, to ultimately provide guidance to the clinician with regard to appropriate testing and therapeutics.

\section{Materials and methods}

\section{Samples}

A search of adenosquamous carcinoma was conducted by natural language search of the pathology 
database for 'adenosquamous' and 'squamous' among lung resection specimen classes from 1997 to 2011. A total of 26 cases were identified as adenosquamous by report, and all slides from these cases were reviewed. In total, 19 cases met morphological criteria for adenosquamous carcinoma, and in controversial cases, immunohistochemistry for thyroid transcription factor 1, p63, and cytokeratin $5 / 6$ as well as mucicarmine stains were performed to confirm the diagnosis. Squamous carcinoma $(n=17)$ and adenocarcinoma $(n=121)$ cases were obtained from tissue microarrays that were described previously. ${ }^{20,21}$ There were 121 adenocarcinomas and 17 squamous cell carcinomas. Each tissue was formalin-fixed, paraffin-embedded, and then used for immunohistochemistry analysis. All analyses were conducted on the surgically resected specimens, from which the pathologist chose a representative area of tumor. All tumor specimens were pathologically reviewed and classified using the World Health Organization lung tumor classification scheme. The study was approved by the hospital's Institutional Review Board.

\section{Measurement and Quantification of Thymidylate Synthase Levels}

From each paraffin block, sections were prepared and stained with hematoxylin and eosin for conventional histological examination. In addition, whole-slide specimens and tissue microarrays were stained for thymidylate synthase expression by immunohistochemistry using an anti-thymidylate synthase antibody (Life Technologies Clone 106, Grand Island, NY, USA). Two independent investigators assessed the intensity of the staining by the intensity score, a number ranging from 0 to 2 , where 0 was no detectable staining, 1 for low-intensity staining, and 2 for strong staining. The percentage of cells that stained positive were also scored. An $\mathrm{H}$-score (range 0-200) was generated, based on the cross-product of the intensity score and the percentage of positive cells. This was performed for all cases. In addition, for the adenosquamous samples, squamous and glandular components were separately assessed using the same histological scoring system.

\section{Microdissection and Mutation Status}

All adenosquamous specimens were assessed for EGFR and KRAS mutations. Manual needle dissection to enrich for tumor cells was performed on deparaffinized histological sections stained with cresyl violet. DNA was extracted using the QIAamp Formalin-Fixed Paraffin-Embedded Tissue Kit (Qiagen, Valencia, CA, USA). Mutation for EGFR and KRAS was detected using Sanger sequencing. For cases with mutation, a separate round of manual microdissection was performed to separately
Table 1 Baseline characteristics of adenosquamous patients

\begin{tabular}{|c|c|c|}
\hline Characteristic & Number & Percentage \\
\hline \multicolumn{3}{|c|}{ Age (median, 72 years range $45-83$ years) } \\
\hline$<$ Median & 9 & 47 \\
\hline$\geq$ Median & 10 & 53 \\
\hline \multicolumn{3}{|l|}{ Gender } \\
\hline Male & 10 & 53 \\
\hline Female & 9 & 47 \\
\hline \multicolumn{3}{|l|}{ Stage } \\
\hline IA & 5 & 26 \\
\hline IB & 3 & 16 \\
\hline IIA & 4 & 21 \\
\hline IIB & 4 & 21 \\
\hline IIIA & 3 & 16 \\
\hline
\end{tabular}

dissect squamous carcinoma from adenocarcinoma areas. Manual needle dissection was performed using an Olympus BX40 microscope at $\times 50$ magnification with a vacuum-driven collection system for sample collection from the slide. Areas for dissection were chosen guided by morphology and immunohistochemistry for thyroid transcription factor 1 and p63 on corresponding serial section. Only characteristic areas of the two histologies of the tumor were chosen for dissection in this phase of the study.

\section{Statistics}

One-way ANOVA was conducted to compare the mean values of the three groups: squamous cell carcinoma, adenocarcinoma, and adenosquamous carcinoma. Tukey's multiple comparison test was used for pairwise comparison for the three different pairing combinations. To assess whether TS expression was correlated within an adenosquamous specimen, a permutation method was used. To determine the statistical significance, the actual correlation coefficient was compared with the correlation coefficient of 10000 random permutations of the data set.

\section{Results}

\section{Demographics of Adenosquamous Carcinoma Cases}

Out of 19 adenosquamous patients, 53\% were men and $47 \%$ women. Age ranged from 45 to 83 years old, with a median age of 72 . The stages ranged from IA-IIIA. Table 1 demonstrates the baseline tumor characteristics.

\section{Overall TS Expression}

Adenocarcinomas $(n=121)$ ranged in intensity from 0 to 2, with 45 with a score of 0,54 with a score of 1 , and 22 with a score of 2 . The percentage of cells stained ranged from 0 to $90 \%$. All four 


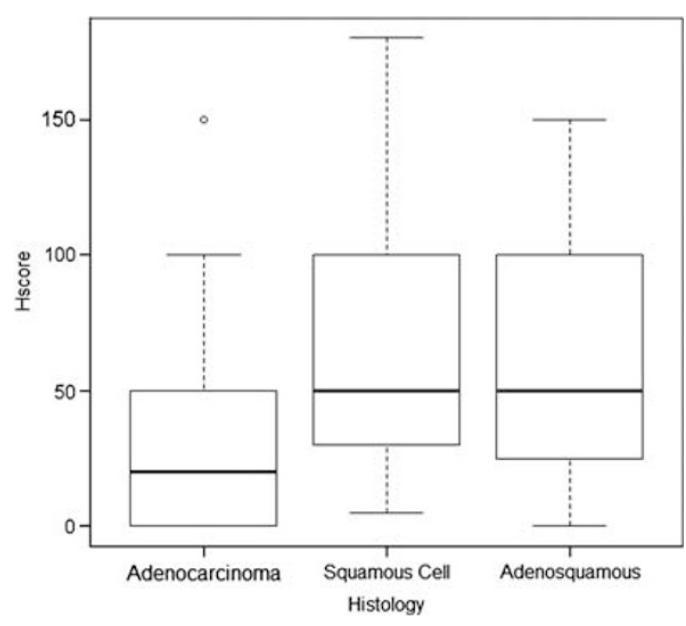

Figure 1 Thymidylate synthase staining expressed as H-score. Horizontal lines in the middle represent median values, and upper and lower bars represent the distance from the 10th to 90th percentile from the median, respectively.

adenocarcinomas that stained $90 \%$ of cells had intensities of 1, and out of the 18 that stained $75 \%$, and 16 had intensities of 1 with only 2 with intensities of 2 . The average $\mathrm{H}$-score of thymidylate synthase expression was 32.5 (95\% confidence interval (CI), 25.6-39.0).

Squamous cell carcinomas $(n=17)$ ranged in intensity from 1 to 2 , 8 with a score of 1 , and 9 with a score of 2 . The percentage stained ranged from 5 to $90 \%$. The average $\mathrm{H}$-score of thymidylate synthase expression was 66.5 (95\% CI, 41.1-91.8).

Finally, adenosquamous carcinoma samples $(n=19)$ were assessed. They ranged in intensity from 0 to 2 , with 1 with a score of 0,10 with a score of 1 , and 7 with a score of 2 . Percentage stained ranged from 0 to $100 \%$. The average H-score of thymidylate synthase expression was 64.7 (95\% CI, 41.6-87.8). Overall, the mean expression levels for adenocarcinoma, adenosquamous carcinoma, and squamous cell carcinoma were $32.5,64.7$, and 66.5 , respectively, $(P=0.0001$; Figure 1 , Table 2$)$. Figure 2 demonstrates examples of immunohistochemistry staining with scores $0-2$.

Tukey's multiple comparison adjustment test was performed for pairwise comparison. There was a significant difference between the mean $\mathrm{H}$-score values of the adenocarcinoma and the squamous cell carcinoma group $(P$-value $=0.004)$. There was also a significant difference between the mean values of the adenocarcinoma and the adenosquamous group $(P$-value $=0.004)$. However, the difference between the mean values of the squamous cell and the adenosquamous group was not significant $(P$-value $=0.99)$.

\section{TS Expression in Adenosquamous Samples}

Each adenosquamous tumor was then reevaluated by independent evaluation of the squamous and the
Table 2 Summary statistics of thymidylate synthase staining expressed as an $\mathrm{H}$-score in adenocarcinomas, adenosquamous carcinomas, and squamous cell carcinomas

\begin{tabular}{lccccccc}
\hline & Mean Median Min Max & $\begin{array}{c}\text { 10th } \\
\text { Percen- } \\
\text { tile }\end{array}$ & $\begin{array}{c}\text { 90th } \\
\text { Percen- } \\
\text { tile }\end{array}$ \\
\hline $\begin{array}{l}\text { Adenocarcinoma } \\
\text { Adenosquamous }\end{array}$ & 32.31 & 20 & 0 & 150 & 0 & 75 \\
Squamous & 66.74 & 50 & 0 & 150 & 5 & 138 \\
& 66.47 & 50 & 5 & 180 & 8 & 120 \\
\hline
\end{tabular}

glandular components. The glandular components ranged in intensity from 0 to 2 and stained from 5 to $100 \%$. The squamous components ranged in intensity from 0 to 2 and stained from 0 to $100 \%$. The mean $\mathrm{H}$-scores for the two components were 53.4; 95\% CI, 27.5-79.3 for glandular, and 61.6; 95\% CI, 41.6-81.5 for squamous.

The correlation coefficient between the squamous and glandular components was 0.48. Based on the permutation method, this was more closely correlated than would be expected by random chance alone (permutation $P$-value $<0.05$ ), and thymidylate synthase expression was higher than the adenocarcinoma group.

\section{Mutational Status}

Out of 19 adenosquamous specimens, there were 2 with EGFR mutations (11\%). In all, 6 of 18 (one failure) harbored KRAS mutations (33\%), with 5 of 6 being transversion mutations. All mutations were mutually exclusive. Upon microdissection, all of the mutated specimens revealed their specific mutations in both the glandular and squamous cell component (one specimen could not be accurately dissected). Table 3 demonstrates the specific mutations for each adenosquamous specimen.

The mean H-score for thymidylate synthase expression for the mutated specimens was 60 , as compared with the overall $\mathrm{H}$-score for adenosquamous specimens of 64.7 .

\section{Discussion}

In this study, we set out to characterize thymidylate synthase expression and molecular status in adenosquamous carcinomas of the lung. As mentioned before, over the last few years, there has been a considerable revolution in treatment of non-small cell lung cancer. Our knowledge of the molecular basis of these tumors, as well as our greater understanding of markers for risk stratification, has changed treatment from standard doublet chemotherapy without consideration to histology, to more targeted approach. EGFR mutations and EML4/ $A L K$ translocations, for example, now indicate a different route for treatment. On the contrary, KRAS mutations have been shown to be negative 

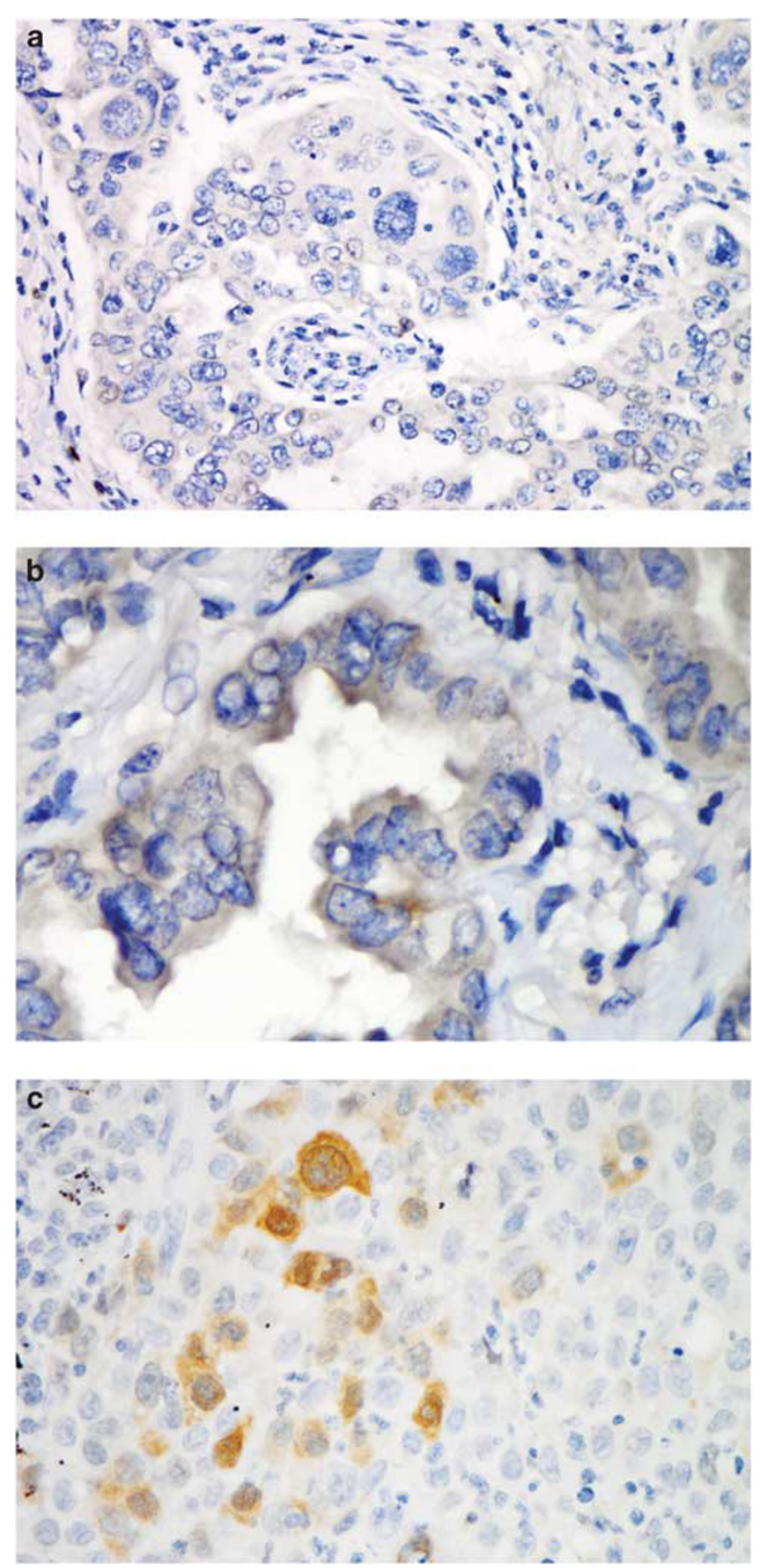

Figure 2 Thymidylate synthase immunohistochemistry. (a) Negative stain (score 0) in a poorly differentiated adenocarcinoma. (b) $1+$ positive staining in an adenocarcinoma. (c) $2+$ positive staining in a squamous cell carcinoma (diaminobenzidine immunohistochemistry, original magnification (a, c $\times 100 ; \mathbf{b} \times 150)$.

predictors of response to single-agent EGFR TKIs in advanced non-small cell lung cancer. ${ }^{22,23}$ Selection of chemotherapeutic agents is also targeted, and pemetrexed has been shown to be more effective in adenocarcinomas than squamous cell carcinomas, and it is believed that this is dependent on the levels of thymidylate synthase expression. ${ }^{16-18}$

Unfortunately, although more options now exist for patients with non-small cell lung cancer, mixed
Table 3 Summary of EGFR and KRAS mutations in the adenosquamous specimens, including the microdissection cases

\begin{tabular}{|c|c|c|c|c|}
\hline \multirow[t]{2}{*}{ Specimen } & \multirow[t]{2}{*}{$\begin{array}{l}\text { EGFR } \\
\text { status }\end{array}$} & \multirow[t]{2}{*}{$\begin{array}{l}\text { KRAS } \\
\text { status }\end{array}$} & \multicolumn{2}{|c|}{$\begin{array}{l}\text { Microdissection } \\
\text { mutation status }\end{array}$} \\
\hline & & & Adenosquamous & Squamous \\
\hline 1 & WT & WT & Not performed & $\begin{array}{l}\text { Not } \\
\text { performed }\end{array}$ \\
\hline 2 & WT & WT & Not performed & $\begin{array}{l}\text { Not } \\
\text { performed }\end{array}$ \\
\hline 3 & WT & $\begin{array}{l}\text { Unable to } \\
\text { obtain }\end{array}$ & Not performed & $\begin{array}{l}\text { Not } \\
\text { performed }\end{array}$ \\
\hline 4 & WT & WT & Not performed & $\begin{array}{l}\text { Not } \\
\text { performed }\end{array}$ \\
\hline 5 & L858R & WT & L858R & L858R \\
\hline 6 & WT & Gly12Cys & Gly12Cys & Gly12Cys \\
\hline 7 & WT & WT & Not performed & $\begin{array}{l}\text { Not } \\
\text { performed }\end{array}$ \\
\hline 8 & WT & Gly12Val & Gly12Val & Gly12Val \\
\hline 9 & WT & WT & Not performed & $\begin{array}{l}\text { Not } \\
\text { performed }\end{array}$ \\
\hline 10 & WT & Gly12Cys & Gly12Cys & Gly12Cys \\
\hline 11 & L858R & WT & L858R & L858R \\
\hline 12 & WT & WT & Not performed & $\begin{array}{l}\text { Not } \\
\text { performed }\end{array}$ \\
\hline 13 & WT & WT & Not performed & $\begin{array}{l}\text { Not } \\
\text { performed }\end{array}$ \\
\hline 14 & WT & Gly12Asp & Unable to obtain & $\begin{array}{l}\text { Unable to } \\
\text { obtain }\end{array}$ \\
\hline 15 & WT & WT & Not performed & $\begin{array}{l}\text { Not } \\
\text { performed }\end{array}$ \\
\hline 16 & WT & WT & Not performed & $\begin{array}{l}\text { Not } \\
\text { performed }\end{array}$ \\
\hline 17 & WT & Gly12Val & Gly12Val & Gly12Val \\
\hline 18 & WT & WT & Not performed & $\begin{array}{l}\text { Not } \\
\text { performed }\end{array}$ \\
\hline 19 & WT & Gly12Cys & Gly12Cys & Gly12Cys \\
\hline
\end{tabular}

histology tumors such as adenosquamous carcinomas have not yet been extensively studied, and no clear treatment protocols exist. This is especially disconcerting, as adenosquamous carcinomas have been shown to have poorer prognosis than adenocarcinomas and squamous cell carcinomas in many studies, ${ }^{4,5}$ including Takamori et al ${ }^{24}$ of 56 adenosquamous carcinomas, 1 of the largest adenosquamous series, in which they compared Kaplan-Meier survival curves of these three different types of non-small cell lung cancer. They noted poor survival even among stages I and II adenosquamous carcinomas, but stages III and IV adenosquamous carcinomas still retained the poorer prognoses. Given this observation, chemotherapy and targeted therapeutic considerations are especially important.

Thus, we hoped to further understanding of adenosquamous carcinomas. The data from this study indicate significantly higher thymidylate synthase expression levels in squamous cell carcinomas than adenocarcinomas, consistent with prior studies (using both immunohistochemistry and direct mRNA measurement ${ }^{15,25}$ ) regarding histology and thymidylate synthase. This study also identifies high thymidylate synthase expression in 
adenosquamous tumors, showing more similarity to squamous cell specimens than adenocarcinomas. Interestingly, the two components of the adenosquamous tumors appear to be correlated, suggesting that the expression of thymidylate synthase is driven more by other tumor determinants than histological pattern. This is also consistent with the Kanazawa study $^{26}$ of 12 adenosquamous tumors, in which they sought to determine polyclonality versus monoclonality of these tumors. With immunohistochemistry, they found higher expression levels of squamous cell carcinoma-related antigen and lower expression of Mucin-1 in the glandular components than usual adenocarcinomas of the lung. They also found that the immunoreactivity of $p 53$ was consistent in both components. All these results led them to conclude that adenosquamous carcinomas derive from a monoclonal transition from squamous cell carcinoma to adenocarcinoma, and not a polyclonal pathway. Bastide et $a l^{27}$ have since performed a comparative transcriptome analysis of adenosquamous, squamous, and adenocarcinomas in rats, and found specific gene signatures for each tumor type, concluding that adenosquamous carcinomas are not simply a mix of adenocarcinomas and squamous cell carcinomas.

To further examine this conclusion, we examined KRAS and EGFR mutational status for all of our adenosquamous samples. In the literature, the prevalence of KRAS mutations in adenocarcinomas is roughly $22 \%^{28}$ and EGFR mutations in adenocarcinomas is roughly $10-15 \%, 29$ with both being mutually exclusive. Interestingly, our 19 adenosquamous specimens showed an EGFR mutation rate of $11 \%$ and a KRAS mutation rate of $33 \%$, which varies from Kanazawa and Tochigi's studies but is consistent with the adenocarcinoma literature. In all of our microdissected specimens, there was concordance of the mutation in both components of the tumor. This is particularly interesting as KRAS and EGFR mutations are seldom seen in squamous cell carcinomas. Tochigi et $a l^{19}$ also performed a microdissection study of adenosquamous carcinomas, and out of 23 cases, they found 3 with EGFR mutations, with 2 of them having the identical mutation in the glandular and squamous components. They also had three KRAS mutations, which were seen in both components. Also of note, the mean H-score in the mutated samples was 60 (compared with adenocarcinoma of 32); while there were only 8 mutated samples and not sufficient power to rule out a significant difference, these data suggest that the thymidylate synthase results are independent of mutation status. That is, the mutated adenosquamous specimens do not seem to act more like adenocarcinomas with regard to this parameter, despite the mutational status. Again, all of this information leads us to believe that adenosquamous carcinomas are not simple mixtures of their two histological tumor counterparts, but instead are much more complex.
Although it is difficult to draw clinical conclusions from a histological project, some possible extrapolations can be made. Given that thymidylate synthase is the main target of pemetrexed and given the similarity of thymidylate synthase expression between squamous cell and adenosquamous carcinomas, it is possible that pemetrexed may also have inferior clinical activity in adenosquamous carcinomas. The next step will be a retrospective review of adenosquamous patients versus adenocarcinoma patients who have received pemetrexed, anticipating a significantly lower response rate in the adenosquamous patients. Indeed, if thymidylate synthase expression determines responsiveness to pemetrexed, then based on the wide range of thymidylate synthase expression levels in all histotypes, it is conceivable that in the future, patient selection for pemetrexed may be better determined based on the tumor's level of enzymatic expression of thymidylate synthase instead of relying on the surrogate marker of histology.

An additional pitfall highlighted by this study is the diagnosis of squamous histology on small samples. The morphology and staining of squamous carcinoma and of squamous component of adenosquamous carcinoma are identical; however, mutational study has the potential to reveal EGFR or KRAS mutations on a small sample demonstrating only squamous component of an adenosquamous carcinoma. A recent study by Rekhtman et $a l^{30}$ showed that pure squamous carcinomas, once carefully classified, very rarely harbor EGFR or KRAS mutation. Although an uncommon tumor type, a small sample of an adenosquamous carcinoma that reveals only the squamous component may be revealed to be more likely adenosquamous carcinoma through molecular testing.

One of the limitations of our study is the subjective nature of immunohistochemical scoring. The samples were graded by two independent scorers to minimize this bias but admittedly, the scorers could not be blinded to the histology while scoring. However, the consistent staining of the glandular component in adenosquamous tumors is contrary to this bias. Another limitation is the small sample size of adenosquamous tumors; this is owing to the rare nature of this histological subtype and in fact represents one of the larger well-characterized collections in the literature.

In conclusion, this study clearly shows a significant difference in thymidylate synthase expression through immunohistochemistry in adenocarcinoma, adenosquamous carcinoma, and squamous cell carcinoma. Adenosquamous carcinomas and squamous cell carcinomas appear to be more similar for this parameter than pure adenocarcinomas, despite the molecular similarity between adenosquamous and adenocarcinoma. When looking at the individual components of the adenosquamous tumors, they are more closely correlated for thymidylate synthase level than expected by random chance. 
The molecular markers showed convergence in the microdissected samples, confirming prior observations of a clonal origin for these oncogenic driver mutations. Future studies will examine these findings from a clinical standpoint. Also, tissue collections of adenosquamous tumors such as ours will provide further opportunities to better understand the genetic basis of the histological divergence of these tumors based on studying key transcription factors, such as thyroid transcriptional factor 1 and SRY (sex-determining region Y)-box 2, as well as other oncogenic drivers of squamous cell carcinoma formation, such as fibroblast growth factor receptor 1 and discoidin death receptor 2 .

\section{Acknowledgements}

This work was supported by a grant from the American Cancer Society (Grant no. RSG-08-30301-TBE to BH).

\section{Disclosure/conflict of interest}

The authors declare no conflict of interest.

\section{References}

1 Siegel R, Ward E, Brawley O, et al. Cancer statistics, 2011: the impact of eliminating socioeconomic and racial disparities on premature cancer deaths. CA Cancer J Clin 2011;61:212-236.

2 Travis WD, Travis LB, Devesa SS. Lung cancer. Cancer 1995;75:191-202.

3 Gibbs AR, Thunnissen FB. Histological typing of lung and pleural tumours: third edition. J Clin Pathol 2001; 54:498-499.

4 Hsia JY, Chen CY, Hsu CP, et al. Adenosquamous carcinoma of the lung. Surgical results compared with squamous cell and adenocarcinoma. Scand Cardiovasc J 1999;33:29-32.

5 Nakagawa K, Yasumitu T, Fukuhara K, et al. Poor prognosis after lung resection for patients with adenosquamous carcinoma of the lung. Ann Thorac Surg 2003;75:1740-1744.

6 Johnson DH, Fehrenbacher L, Novotny WF, et al. Randomized phase II trial comparing bevacizumab plus carboplatin and paclitaxel with carboplatin and paclitaxel alone in previously untreated locally advanced or metastatic non-small-cell lung cancer. J Clin Oncol 2004;22:2184-2191.

7 Sandler A, Gray R, Perry MC, et al. Paclitaxelcarboplatin alone or with bevacizumab for non-smallcell lung cancer. N Engl J Med 2006;355:2542-2550.

8 Mok TS, Wu YL, Thongprasert S, et al. Gefitinib or carboplatin-paclitaxel in pulmonary adenocarcinoma. N Engl J Med 2009;361:947-957.

9 Maemondo M, Inoue A, Kobayashi K, et al. Gefitinib or chemotherapy for non-small-cell lung cancer with mutated EGFR. N Engl J Med 2010;362:2380-2388.

10 Kwak EL, Bang YJ, Camidge DR, et al. Anaplastic lymphoma kinase inhibition in non-small-cell lung cancer. N Engl J Med 2010;363:1693-1703.
11 Schultz RM, Patel VF, Worzalla JF, et al. Role of thymidylate synthase in the antitumor activity of the multitargeted antifolate, LY231514. Anticancer Res 1999;19:437-443.

12 Hanna N, Shepherd FA, Fossella FV, et al. Randomized phase III trial of pemetrexed versus docetaxel in patients with non-small-cell lung cancer previously treated with chemotherapy. J Clin Oncol 2004;22: 1589-1597.

13 Scagliotti GV, Parikh P, von Pawel J, et al. Phase III study comparing cisplatin plus gemcitabine with cisplatin plus pemetrexed in chemotherapy-naive patients with advanced-stage non-small-cell lung cancer. J Clin Oncol 2008;26:3543-3551.

14 Ciuleanu T, Brodowicz T, Zielinski C, et al. Maintenance pemetrexed plus best supportive care versus placebo plus best supportive care for non-small-cell lung cancer: a randomised, double-blind, phase 3 study. Lancet 2009;374:1432-1440.

15 Ceppi P, Volante M, Saviozzi S, et al. Squamous cell carcinoma of the lung compared with other histotypes shows higher messenger RNA and protein levels for thymidylate synthase. Cancer 2006;107:1589-1596.

16 Bepler G, Sommers KE, Cantor A, et al. Clinical efficacy and predictive molecular markers of neoadjuvant gemcitabine and pemetrexed in resectable nonsmall cell lung cancer. J Thorac Oncol 2008;3: 1112-1118.

17 Ozasa H, Oguri T, Uemura T, et al. Significance of thymidylate synthase for resistance to pemetrexed in lung cancer. Cancer Sci 2010;101:161-166.

18 Chang $\mathrm{MH}$, Ahn JS, Lee J, et al. The efficacy of pemetrexed as a third- or fourth-line therapy and the significance of thymidylate synthase expression in patients with advanced non-small cell lung cancer. Lung Cancer 2010;69:323-329.

19 Tochigi N, Dacic S, Nikiforova M, et al. Adenosquamous carcinoma of the lung: a microdissection study of KRAS and EGFR mutational and amplification status in a western patient population. Am J Clin Pathol 2011;135:783-789.

20 Borczuk AC, Gorenstein L, Walter KL, et al. Non-smallcell lung cancer molecular signatures recapitulate lung developmental pathways. Am J Pathol 2003;163: 1949-1960.

21 Borczuk AC, Qian F, Kazeros A, et al. Invasive size is an independent predictor of survival in pulmonary adenocarcinoma. Am J Surg Pathol 2009;33:462-469.

22 Linardou H, Dahabreh IJ, Kanaloupiti D, et al. Assessment of somatic k-RAS mutations as a mechanism associated with resistance to EGFR-targeted agents: a systematic review and meta-analysis of studies in advanced non-small-cell lung cancer and metastatic colorectal cancer. Lancet Oncol 2008;9:962-972.

23 Eberhard DA, Johnson BE, Amler LC, et al. Mutations in the epidermal growth factor receptor and in KRAS are predictive and prognostic indicators in patients with non-small-cell lung cancer treated with chemotherapy alone and in combination with erlotinib. J Clin Oncol 2005;23:5900-5909.

24 Takamori S, Noguchi M, Morinaga S, et al. Clinicopathologic characteristics of adenosquamous carcinoma of the lung. Cancer 1991;67:649-654.

25 Tanaka F, Wada H, Fukui Y, et al. Thymidylate synthase (TS) gene expression in primary lung cancer patients: a large-scale study in Japanese population. Ann Oncol 2011;22:1791-1797. 
26 Kanazawa H, Ebina M, Ino-Oka N, et al. Transition from squamous cell carcinoma to adenocarcinoma in adenosquamous carcinoma of the lung. Am J Pathol 2000;156:1289-1298.

27 Bastide K, Ugolin N, Levalois C, et al. Are adenosquamous lung carcinomas a simple mix of adenocarcinomas and squamous cell carcinomas, or more complex at the molecular level? Lung Cancer 2010;68:1-9.

28 Riely GJ, Marks J, Pao W. KRAS mutations in nonsmall cell lung cancer. Proc Am Thorac Soc 2009;6: 201-205.
29 Marchetti A, Martella C, Felicioni L, et al. EGFR mutations in non-small-cell lung cancer: analysis of a large series of cases and development of a rapid and sensitive method for diagnostic screening with potential implications on pharmacologic treatment. J Clin Oncol 2005;23:857-865.

30 Rekhtman N, Paik PK, Arcila ME, et al. Clarifying the spectrum of driver oncogene mutations in biomarkerverified squamous carcinoma of lung: lack of EGFR/ KRAS and presence of PIK3CA/AKT1 mutations Clin Cancer Res 2012;18:1167-1176. 